\title{
Diversidad florística y conservación del Santuario Regional de Piura Manglares San Pedro de Vice-Sechura
}

\author{
Floristic diversity and conservation of the Regional Sanctuary of Piura \\ Manglares San Pedro of Vice-Sechura
}

\author{
Manuel Charcape-Ravelo ${ }^{1}$ y Fanny Moutarde ${ }^{2}$
}

Publicación: 08/12/2005

\begin{abstract}
Resumen
El presente trabajo se llevó a cabo con la finalidad de conocer la composición de las fanerógamas del Santuario Regional de Piura Manglares San Pedro de Vice-Sechura. Su importancia radica en que es el manglar más austral del Pacífico de Sudamérica. Dicho trabajo fue realizado desde abril del 2000 hasta febrero del 2004. Para la realización del presente estudio se realizaron visitas periódicas cada dos meses al lugar, se colectaron las plantas bajo procedimientos estándar. Las muestras se determinaron en el lugar y algunas fueron llevadas al laboratorio de Botánica de la Universidad Nacional de Piura y al laboratorio de Botánica Fanerogámica de la Universidad Nacional de Trujillo para su determinación definitiva; luego, las muestras se depositaron en el herbario en formación de la Universidad Nacional de Piura. El manglar consiste en primer lugar de árboles y arbustos de Avicennia germinans (L.) L. (Verbenaceae) «mangle prieto». En el presente inventario se documentan un total de 44 especies de fanerógamas correspondiendo a 36 géneros en 21 familias para el manglar.
\end{abstract}

Palabras clave: Manglar, Diversidad Florística, Piura, Perú, Conservación.

\section{Abstract}

The present work was carried out with the aim to document the composition of the flowering plants of the Regional Sanctuary of Piura «Manglares San Pedro», of Vice-Sechura. Its importance resides in that it is the most southern mangrove forest in the Pacific of South America. The study was carried out from April of the 2000 until February of the 2004. For the realization of the present work periodic visits every two months to the place were undertaken, the plants were collected under the standard procedures. The samples were determined in the place and some were taken to the laboratory of Botany of the National University of Piura and the laboratory of Phanerogamic Botany of the National University of Trujillo for their definitive determination, then the samples were deposited in the Herbarium in formation of the National University of Piura. The mangrove forest primarily consists of trees and shrubs of Avicennia germinans (L.) L. (Verbenaceae) «mangle prieto». The present inventory reports 44 species of phanerogams belonging to 36 genera in 21 families from the Mangrove.

Keywords: Mangrove, Floristic diversity, Piura, Peru, Conservation.

\section{Introducción}

Los humedales comprenden una gran variedad de ecosistemas, por lo cual su definición es en general compleja. Aunque existen varias definiciones de humedales, la más utilizada en la actualidad es la definición de la

${ }^{1}$ Universidad Nacional de Piura, Departamento Académico de Ciencias Biológicas y Ecología, Desarrollo Humano y Conservación en el Perú (EDHUCO-PERU).

E-mail: edhuco_peru@yahoo.com

${ }^{2}$ Université Paris 1. Panthéon. Sorbonne - Francia.

E-mail: manuelbot@yahoo.com convención de Ramsar: «Extensiones de marismas, pantanos, turberas o aguas de régimen natural o artificial, permanentes o temporales, estancadas o corrientes, dulces, salobres o saladas, incluyendo las extensiones de agua marina cuya profundidad en marea baja no exceda de seis metros» (Ramsar, 1990; Tabilo, 1999).

Aunque existen varias clasificaciones de humedales, hemos creído conveniente hacer uso de las propuestas por Bravo \& Windevoxhel (1997) y la de Dugan (1992), debido a la buena adaptación del manglar a estas clasificaciones, el cual cae dentro de la 
clasificación de Sistema Estuarino, que se define como, ambientes costeros que tienen conexión con mar abierto. Los manglares son comunidades de plantas leñosas que crecen en las zonas de mareas, en agua salada, cuya concentración es de 35\% aproximadamente (presión osmótica potencial de 25 atmósferas) de lo que se deduce fácilmente que se trata de plantas altamente resistentes a la salinidad del hábitat. Comprenden aproximadamente unas 20 especies de mangles. Es importante distinguir entre los manglares orientales, los cuales son más ricos en especies que los manglares occidentales. Ambos tipos son, pues, comunidades de climas cálidos o tropicales, donde el litoral es plano, fangoso, de aguas tranquilas y salobres, por lo que penetran algunas veces hacia el interior de los continentes, siguiendo las riberas fluviales donde se detienen cuando el agua deja de ser salobre (Mostacero et al., 1996; Tabilo, 1999; WRM, 2002).

El sistema ecológico de los manglares se encuentra en las zonas tropicales de África, América, Asia y Oceanía. En realidad, se trata de un bosque tropical que se desarrolla en la costa, principalmente donde hay deltas importantes de ríos, que desembocan en el mar, donde se producen acumulaciones de fango como sustrato y variaciones permanentes de salinidad. El manglar es un macrocosmo de abundantes formas de vida, tanto de flora como de fauna. Además sirve de hábitat para muchos animales en forma permanente y/o temporal, así como sitio de descanso y alimentación a animales migratorios por motivo de cambio de estaciones climáticas. Hay que mencionar que dentro de los ecosistemas relacionados con los ambientes marinos, los manglares ocupan el segundo lugar en producción de biomasa y energía, después de los arrecifes de coral. Su gran diversidad se debe a la interacción de las aguas dulce y salada que convergen para formar los estuarios, y el manglar San Pedro no escapa a esta realidad (Peña, 1970; Mostacero et al., 1996; Cambra, 2001; Charcape et al., 2003).

El bosque que forma el manglar está constituido en su mayor parte por árboles de mangle, plantas especializadas en soportar las ele- vadas concentraciones salinas y condiciones adversas de las zonas costeras y que en su conjunto forman la asociación vegetal llamada manglar. Además, por la rapidez con que son descompuestos los materiales que mueren dentro del manglar, permite que éstos se puedan incorporar como nutrientes al resto de organismos que lo habitan. En América los manglares se distribuyen desde el Trópico de Cáncer hasta $\operatorname{los} 3^{\circ} 30^{\prime} \mathrm{S}$ en el Océano Pacífico, deteniéndose allí, debido al sistema de corrientes del Perú. Igualmente se distribuyen desde aproximadamente los $30^{\circ} \mathrm{N}$ hasta los $25^{\circ} \mathrm{S}$ (Florianópolis, Brasil), en el Atlántico (Peña, 1970; ONERN, 1977; Mostacero et al., 1996; CDC-UNALM, 1992).

Los manglares en el Perú son complejos boscosos con una biota característica constituida por especies de mangle de los géneros Rhizophora, Avicennia, Laguncularia y Conocarpus; se distribuyen desde Tumbes, límite con el Ecuador, hasta San Pedro (ViceSechura, Piura; límite sur, para este ecosistema en el Pacífico americano). Se caracterizan por mantener una fauna endémica, tales como la concha negra Anadara spp; los cangrejos violinistas $U c a$ spp; el cangrejo gigante Ucides spp.; caracoles Cerithidea y Nassarius; el caracol coco Melongena patula; las ostras Ostrea spp. (Rodríguez et al., 1997; CDCUNALM, 1992, 1997; Charcape et al. 2003).

El manglar San Pedro aún no ha sido bien estudiado. Se conocen pocos trabajos sobre este importante ecosistema por el hecho de estar ubicado en la zona más austral del Pacífico de Sudamérica; su importancia en productividad, su biodiversidad marino-costera, su vulnerabilidad, entre otros, justifica que este ecosistema sea estudiado y conservado.

Con el único afán de conocer la composición de la flora fanerogámica de este manglar, así como el dar a conocer lo que se viene realizando por su conservación, es que se realizó el presente estudio en el último manglar del Pacífico sudamericano. 


\section{Material y Métodos}

El manglar San Pedro de Vice, se ubica en el departamento de Piura, provincia de Sechura, distrito de Vice, entre los $5^{\circ} 30^{\prime} 40^{\prime \prime}$ $5^{\circ} 34^{\prime} 10^{\prime \prime} \mathrm{S}$ y $80^{\circ} 52^{\prime} 50^{\prime \prime}-80^{\circ} 54^{\prime} 45^{\prime \prime} \mathrm{W}$, a $50 \mathrm{~km}$ al suroeste de la ciudad de Piura y a 20 $\mathrm{km}$ al norte de Sechura (Fig. 1). Tiene un área aproximada de 500 ha (GAP, 1998), constituyendo el límite de distribución sur para los manglares en el Pacífico americano. Resulta interesante considerar que el manglar San Pedro empieza a expandirse desde la ocurrencia del evento El Niño 1983.

Las especies consideradas en este trabajo han sido colectadas desde el año 2000. Las exploraciones botánicas se hicieron obedeciendo un cronograma planificado, realizando una visita cada dos meses al lugar, cubriendo recorridos que abarcaron los dos márgenes del río Piura en su desembocadura, desde donde

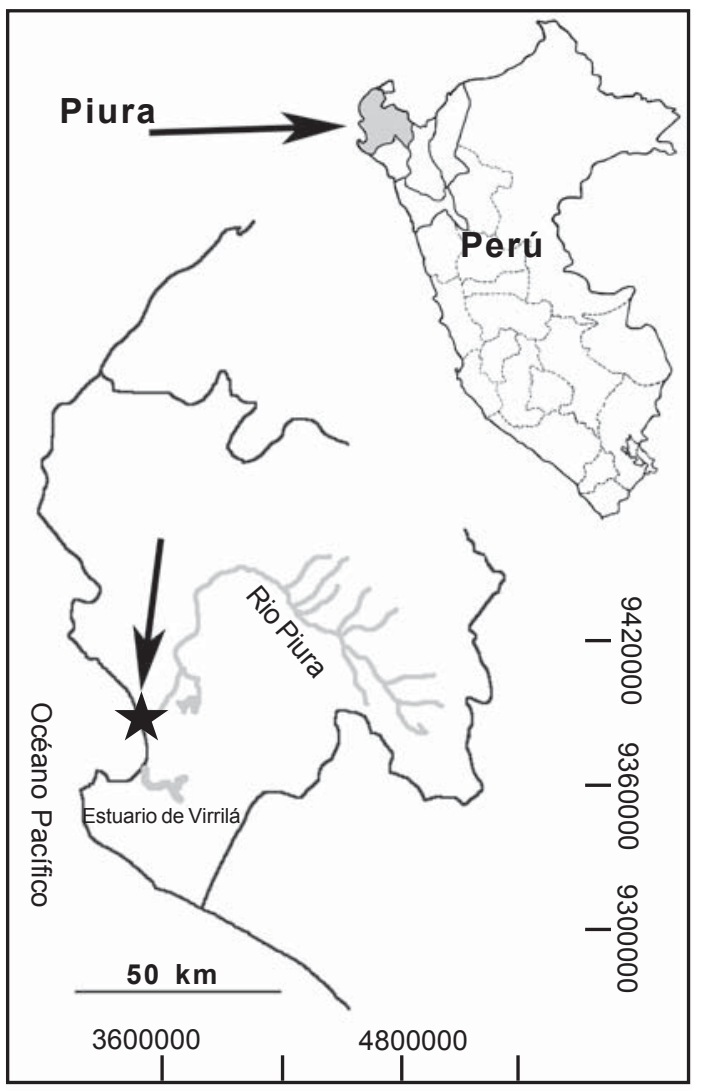

Figura 1. Ubicación del manglar San Pedro de Vice (estrella) en el departamento de Piura. están presentes los primeros mangles a unos 500 metros de la playa San Pedro, hasta donde termina la distribución de los últimos mangles en la provincia de Sechura.

En cada exploración botánica se colectaron las plantas por los procedimientos estándar ayudados de tijeras podadoras y de las prensas botánicas. Se hicieron las respectivas anotaciones, teniendo en cuenta las características taxonómicas, hábito, hábitat, procedencia, distribución altitudinal y latitudinal, épocas de floración y fructificación; utilidad etnobotánica (sobre todo lo referente a lo medicinal), las características edáficas (textura y estructura de los suelos) y algunas características climáticas. Esta información se recabó en un formato previamente establecido, el mismo que servirá para elaborar una base de datos que se utilizará en trabajos posteriores.

El material botánico colectado y posteriormente herborizado, se determinótaxonómicamente in situ; los especímenes que presentaron alguna dificultad en su determinación fueron llevados a los laboratorios de botánica fanerogámica de la Universidades Nacional de Piura y Universidad Nacional de Trujillo, para su determinación definitiva con la ayuda de claves botánicas, bibliografía especializada, flórulas y monografías: J. F. Macbride en su Flora of Peru (1936 a 1986), Vásquez (1997), Gentry (1993); Sagástegui \& Leiva (1993) y Mostacero et al. (2002). Las muestras botánicas colectadas se depositaron en el herbario en formación de la Universidad Nacional de Piura.

En lo referente a su conservación hemos identificado las amenazas que impactan sobre el manglar.

\section{Resultados}

\section{Diversidad y ecología}

Fueron inventariadas un total de 44 especies de fanerógamas, comprendidas en la división angisopermae, 21 familias y 36 géneros; siendo el mangle prieto, Avicennia germinans (L.) L. (Verbenaceae), el biotipo más conspicuo. El listado completo se encuen- 
Tabla 1. Lista alfabética de las fanerógamas del Santuario Regional «Manglares San Pedro» de Vice (Sechura, Piura).

\begin{tabular}{|c|c|c|}
\hline Nombre científico & Familia & Nombre común \\
\hline Acacia huarango Ruiz ex J. F. Macbride & Fabaceae & «huarango» \\
\hline Acacia macracantha H. \& B. ex Willdenow & Fabaceae & «espino», «faique» \\
\hline Aeschynomene tumbezensis J. F. Macbride & Fabaceae & \\
\hline Alternanthera peruviana (Moquin) Suessenguth & Amaranthaceae & «hierba blanca» \\
\hline Alternanthera pungens H.B.K. & Amaranthaceae & «hierba del pollo» \\
\hline Avicennia germinans (L.) L. & Verbenaceae & «mangle prieto» \\
\hline Baccharis salicifolia (R. \& P.) Persoon & Asteraceae & «chilco» \\
\hline Bacopa monnieri (L.) Pennell & Scrophulariaceae & «bacopa» \\
\hline Batis maritima L. & Bataceae & «vidrio» \\
\hline Cenchrus echinatus L. & Poaceae & «cadillo» \\
\hline Cenchrus myosuroides H.B.K. & Poaceae & «cadillo» \\
\hline Chloris virgata Swartz & Poaceae & «grama» \\
\hline Cryptocarpus pyriformis H.B.K. & Nyctaginaceae & «chope» \\
\hline Distichlis spicata (L.) Greene & Poaceae & «grama salada» \\
\hline Exodeconus prostratus (L'Héritier) Raf. & Solanaceae & «campanilla olorosa» \\
\hline Heliotropium curassavicum L. & Boraginaceae & «hierba del alacrán» \\
\hline Indigofera suffruticosa Miller & Fabaceae & «pasto» \\
\hline Ipomoea crassifolia Cavanilles & Convolvulaceae & «bejuco» \\
\hline Laguncularia racemosa (L.) Gaertner f. & Combretaceae & «mangle blanco» \\
\hline Lippia nodiflora (L.) Michaux & Verbenaceae & «turre hembra» \\
\hline Ludwigia peploides (H.B.K.) Raven & Onagraceae & «flor de clavo» \\
\hline Monnina pterocarpa R. \& P. & Polygalaceae & «palomilla» \\
\hline Mutingia calabura L. & Elaeocarpaceae & «cerezo de monte» \\
\hline Parkinsonia aculeata L. & Fabaceae & «azote de Cristo» \\
\hline Passiflora foetida L. & Passifloraceae & «granadilla de culebra» \\
\hline Paspalum bonplandianum Fluegge & Poaceae & "grama» \\
\hline Paspalum racemosum Lamarck & Poaceae & "nudillo» \\
\hline Paspalum vaginatum Swartz & Poaceae & «grama» \\
\hline Phragmites australis (Cavanilles) Trinius ex Steudel & Poaceae & «carricillo» \\
\hline Prosopis juliflora (Swartz) DC. var. horrida (Kunth) Burrkart & Fabaceae & «algarrobo» \\
\hline Prosopis pallida (H. \& B. ex Willdenow) H.B.K. & Fabaceae & «algarrobo» \\
\hline Salicornia fruticosa L. & Chenopodiaceae & «parachique» \\
\hline Scirpus maritimus L. & Cyperaceae & «totora» \\
\hline Sessuvium portulacastrum (L.) L. & Aizoaceae & «lejía verde» \\
\hline Spilanthes leiocarpa DC. & Asteraceae & «turre macho» \\
\hline Sporobulus indicus (L.) R. Brown & Poaceae & «cerillo», «grama» \\
\hline Sporobulus virginicus (L.) Kunth & Poaceae & «grama» \\
\hline Tamarix gallica L. & Tamaricaceae & «ciprés» \\
\hline Tessaria integrifolia R. \& P. & Asteraceae & «pájaro bobo» \\
\hline Tiquilia dichotoma (R. \& P.) Persoon & Boraginaceae & «manito de ratón» \\
\hline Tiquilia paronychioides (Philippi) A. Richardson & Boraginaceae & «flor de arena» \\
\hline Typha angustifolia L. & Typhaceae & «inea», «enea» \\
\hline Vigna adenantha (G. Meyer) Marechal & Fabaceae & «porotillo» \\
\hline Vigna luteola (Jacquin) Bentham & Fabaceae & «porotillo» \\
\hline
\end{tabular}

tra en la Tabla 1. La mayoría de las especies presentes en el manglar son de amplia distribución y presentes en la costa peruana y por sí mismas no representan buena razón para la conservación del manglar. Sin embargo, las dos especies de mangle Laguncularia y Avicennia tienen aquí una población aislada y la más sureña de su distribución en la costa pacífica americana. Respecto a la botánica este hecho es buena razón para esfuerzos de conservación. Más aún, el manglar tiene un rol ecológico muy importante, tanto respecto a la protección de la costa como también de hábitat para un gran número de animales completamente dependientes de este tipo de hábitat. La importancia del manglar, de esta manera, no se basa tanto en su (poca) riqueza botánica, sino en su función ecológica, especialmente respecto a la conservación de la rica fauna del manglar.

\section{Amenazas}

En cuanto a las amenazas identificadas en este manglar, fueron verificadas in situ por los trabajos en campo y también con participación de los mismos pobladores del lugar, las 
mismas que afectan la flora y fauna de la zona; las principales amenazas son:

- Contaminación por el río Piura. Se produce porque las aguas del río reciben el vertido de aguas servidas sin tratamiento, procedentes de la ciudad de Piura y otros pueblos aledaños que lo utilizan como desagüe, vertiendo toda clase de contaminantes; así también en sus orillas se encuentra acumulación de basura dejada por vehículos.

- La industria pesquera también contamina el manglar con sus vertidos en el mar que llegan a varios sectores de la zona.

- La extracción minera en la parte alta del río Piura también trae consigo el peligro de una contaminación seria del manglar.

- Canales de riego, que vierten agua de regadío al río que llega al manglar, esto hace cambiar el grado de salinidad del mismo, pudiendo traer fuertes consecuencias en el mismo.

- Ganadería y destrucción del hábitat. Gente dedicada a la ganadería eventualmente hace su ingreso al manglar y zona aledaña con su ganado, acarreando como consecuencia la destrucción del hábitat; esto también se da por los cerdos ferales que deambulan por el área comiendo y levantando la vegetación.

- Compactación de suelo por vehículos, de todo tamaño, que circulan continuamente muy cerca al manglar, compactando el suelo e impidiendo el desarrollo de nuevas plantas de mangle, así como la perturbación de la fauna del manglar y del anidamiento de las aves.

- Caza furtiva de aves, realizada por gente que mata los pelícanos para aprovechar la carne de la zona del pecho y mata a los flamencos para sacarle las plumas y luego desechan a estos animales.

- Pesca por gente inexperta, es uno de los problemas más graves, pues eventualmente ingresan al manglar y no conocen la extracción de larvas de langostino, destruyendo el bosque de mangle; estas personas extraen las larvas de langostino y peces en forma indiscriminada para comercializarlas, matando especímenes juveniles que aún no están aptos para el consumo. Esta destrucción progresiva está deteriorando el ecosistema y reduciendo el hábitat y alimento de la fauna que mantiene el equilibrio ecológico del manglar. La extracción excesiva de peces y conchas blancas, muchas veces se da empleando redes de arrastre, trayendo consigo un desequilibrio en la trama trófica, que se refleja en la disminución de las especies que es notada por los pescadores artesanales.

- Tala y quema del manglar y del bosque seco (algarrobal) aledaño, estos lugares están siendo impactados por la extracción de madera para leña, dejando sin hábitat y refugio a muchas especies de aves. Sectores del manglar están siendo quemados para ganar terrenos y hacer entradas para la pesca o para construir chozas para los pescadores eventuales, incluso algunas casas muy cercanas al manglar.

- Turismo exagerado, sobre todo en los meses del verano (enero, febrero y marzo), que trae como consecuencia el arrojo de restos de comida, plásticos, emisión de ruidos, música fuerte que ahuyenta a las aves, así como arrojo de restos de basura en gran cantidad.

\section{Conservación}

En últimos años se realizaron varias actividades referidas a la conservación del manglar. Las hemos enfocado desde cuatro ejes principales: educación, capacitación, establecimiento del marco legal y difusión (véase un listado de las actividades en el Apéndice 1). Los dos primeros puntos, educación y capacitación, se viene realizando mediante varios programas para educar y capacitar en primer lugar a los pobladores de Vice, que son los más involucrados con el manglar; y también a la comunidad piurana en general y a otras ciudades del Perú y del extranjero. El marco legal también resulta importante para la conservación del patrimonio natural; en este aspecto, hemos venido asesorando a la municipalidad distrital de Vice y, gracias a los trabajos 
realizados, esta municipalidad y el gobierno regional de Piura tomaron las siguientes acciones legales: el 29 de diciembre del año 2000, mediante resolución municipal 094-MDV, declara a los manglares como "Área de Conservación Municipal». El 07 de agosto de 2003, el gobierno regional de Piura hace lo mismo y declara, junto con el bosque seco aledaño, el reconocimiento como «Santuario Regional de Piura». En cuanto al cuarto punto, difusión, lo venimos realizando en varios medios y eventos. El resultado de todas estas actividades es que ahora la población está mucho más alerta sobre la importancia del manglar y ya tenemos algunos avances respecto a la conservación de este hábitat, en la medida que hemos logrado involucrar tanto al gobierno como a la prensa y a la población en general en este esfuerzo.

\section{Conclusiones}

De las 20 especies de mangles reportadas para el mundo, el manglar San Pedro solamente presenta dos: Avicennia germinans mangle prieto y Laguncularia racemosa mangle blanco, donde el 75\% aproximadamente corresponde a la primera especie, a diferencia de los manglares de Tumbes donde el biotipo característico es Rizophora mangle mangle rojo que no está presente en San Pedro; esto probablemente se deba a las distintas condiciones edafoclimáticas existentes en la zona (Rodríguez et al. 1997; INRENA, 2001; Charcape et al., 2003). Si bien es cierto que el área del manglar San Pedro de Vice es relativamente pequeño (500 ha), comparado con el manglar de Tumbes (2972 ha), es decir, es aproximadamente seis veces más extenso en superficie; sin embargo, hasta el momento hemos encontrado que alberga a 44 especies de fanerógamas, mientras que en el manglar de Tumbes se han reportado 40 especies de fanerógamas, esto debido a su distribución latitudinal (INRENA 2001; Charcape et al., 2003).

Las familias mejor representadas en el manglar San Pedro de Vice son las Poaceae con 10 y Fabaceae con 9 especies respectivamente, la mayoría de las especies vegeta- les son propias de estas zonas, también tenemos especies introducidas como Tamarix gallica (Tamaricaceae), que se han adaptado a este tipo de ecosistemas.

Durante las evaluaciones, hemos podido comprobar que el manglar está siendo fuertemente impactado por personas que acuden al mismo por varios motivos; unos impactan con el tránsito de vehículos de todo tipo que pasan muy cerca a la vegetación y que están compactando el suelo e impiden la propagación de los mangles, asimismo los vehículos causan problemas al perturbar la fauna silvestre presente en la zona. Otro impacto es producido por la tala y quema de los mangles por los pescadores y leñadores, esto a la larga puede traer fuertes consecuencias en la dinámica del bosque y del ecosistema. Sin embargo, los programas de conservación están dando resultados; ellos traen como resultado la toma de dos acciones principales en el marco legal: la municipalidad la denomina Área de Conservación Municipal y el gobierno regional declara la zona como Santuario Regional de Piura.

\section{Literatura citada}

Bravo, J. \& N. Windevoxhel. 1997. Manual para la identificación y clasificación de humedales en Costa Rica. San José, Costa Rica: UICN/ORMA, MINAE, Embajada Real de los Países Bajos.

Cambra Palomino, C. 2002. ¿Réquiem para los manglares? Rev. Ambiente ecológico N. ${ }^{\circ}$ 57. Panamá.

CDC-UNALM. 1992. Estado de conservación de la diversidad natural de la región noroeste del Perú. Lima, Perú: Centro de Datos para la Conservación. Universidad Nacional Agraria La Molina.

Charcape Ravelo, M.; J., Mostacero; E., Vegas; C., Chávez \& R., Barrionuevo. 2003. Fanerógamas del Manglar San Pedro de Vice, Piura-Perú. Libro del II Congreso de la Conservación de la Biodiversidad en los Andes y la Amazonía, y IV Congreso Ecuatoriano de Botánica. Ecuador: Edit. Universidad Técnica Particular de Loja.

Dugan, P. 1992. Conservación de humedales. Un análisis de temas de actualidad y acciones necesarias. Suiza: UICN, Gland.

Engler, A. 1954-1964. Syllabus Der Pflanzenfamilien.

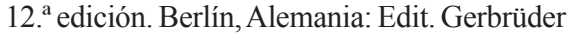
Bortråeger. 
GAP (Grupo de Aves del Perú) y Programa Sostenido De Conservación Y Desarrollo De HumedalesPerú. 1998. Resultados de los Censos Neotropicales de las Aves acuáticas en el Perú. 1992-1995. Lima, Perú.

Gentry, A. 1993. A field guide to the families and genera of woody plants of northwest South America, Colombia, Ecuador and Peru. Washington D.C.: Edit. Conservancy International.

INRENA. 2001. Plan Maestro del Santuario Nacional «Los Manglares de Tumbes. Tumbes, Perú.

Macbride, J.F. 1936-1986. Flora of Peru, vol. XIII, parte I y siguientes. Chicago, EEUU. Edit. Field Musseum of Natural History.

Mostacero L., J.; F. MEJÍA, F. PELÁEZ \& M. CHARCAPE. 1996. Fitogeografía del norte del Perú. Lima, Perú: Edit. Forma e Imagen.

Mostacero, J.; F., Mejía \& O. Gamarra, 2002. Taxonomía de las fanerógamas útiles del Perú. Trujillo, Perú: Edit. Normas Legales S.A.C.

ONERN, 1977. Inventario y evaluación de los recursos naturales de la zona del complejo de Bayovar. Lima, Perú: Oficina Nacional de Evaluación de Recursos Naturales.
Peña, G. 1970. Biocenosis de los manglares Peruanos. Anales Científicos, vol. IX (1 y 2). Lima, Perú.

RAMSAR, 1990. Proceeding of the third Meeting of the conference of the contracting parties. Gland, Suiza: Ramsar Convention Bureau, UICN.

Rodríguez, W.; R., Marcial \& R., Barrionuevo. 1997. Biodiversidad del Manglar de San Pedro entre Mayo 1995-Abril 1997. Sechura. Piura-Perú: UNIVERSALIA.

Sagástegui, A. \& S. Leiva. 1993. Flora invasora de los cultivos del Perú. Trujillo, Perú: Editorial Libertad E I. R. L.

Tabilo Valdivieso, E. 1999. El beneficio de los humedales en América Central: el potencial de los humedales para el desarrollo. 2. ${ }^{\text {a }}$ edición. San José, Costa Rica: Edit. Universidad Nacional Heredia.

Vásquez, M. R. 1997. Flórula de las reservas biológicas de Iquitos - Perú. Monographs in Systematic Botany from the Missouri Botanical Garden, 63: 1-1046.

WRM (World Raiforest Movement) 2002. Manglares, sustento local versus ganancia empresarial. Montevideo, Uruguay.

\section{Apéndice 1}

\section{Actividades referidas a la conservación del manglar realizadas por los autores.}

Curso: Diseño, gestión y ejecución de proyectos en ecología y conservación de la naturaleza. Realizado del 25 al 29 de marzo de 2002 en el mismo manglar, con participación de biólogos y estudiantes de diferentes universidades del Perú y Ecuador. Los capacitadores fueron de Chile y Perú.

Conferencias de sensibilización pro conservación de la naturaleza en Piura, realizado el 16, 19 y 24 de julio de 2002. Organizado por la Universidad Nacional de Piura, Universidad César Vallejo (Piura) y la Asociación Conservacionista Ecología, Desarrollo Humano y Conservación en el Perú (EDHUCO-PERÚ), llevadas a cabo en el auditorio de la Universidad Nacional de Piura.

Curso internacional: Conservación y manejo de los humedales en el Perú, con énfasis en los manglares «San Pedro» de Vice. Realizado del 09 al 11 de enero de 2003, con participación de biólogos y estudiantes de diferentes universidades del Perú y Ecuador. Se llevó a cabo íntegramente en el manglar «San Pedro» de Vice y en el distrito de Vice. Los capacitadores fueron de Chile y Perú.

Curso de capacitación para guías de ecoturismo. Realizado el 11 de enero de 2003, con participación de mototaxistas e interesados del distrito de Vice. Se llevó a cabo en el distrito de Vice. Los capacitadores fueron de Chile, Ecuador y Perú.

Taller: Conservación del manglar «San Pedro» de Vice. Realizado del 13 al 14 de enero de 2003, con participación de la población local, gremios y autoridades del distrito de Vice y distritos colindantes. Se llevó a cabo en el distrito de Vice. Los capacitadores fueron de Chile y Perú.

Curso internacional: Gestión, conservación y manejo de aves playeras migratorias del Pacifico de Sudamérica, realizado del 07 al 10 de enero de 2004, con participación de biólogos y estudiantes de Bolivia, Chile, Ecuador y Perú. Se llevó a cabo en el distrito de Vice y manglar «San Pedro» de Vice. Los capacitadores fueron de Chile y Perú.

Curso internacional: «Gestión, conservación y manejo de aves playeras migratorias y sus hábitat», del 10 al 15 de enero del 2005, en el distrito de Vice y manglar «San Pedro». Capacitadores de Chile y Perú. 


\section{Divulgacion en diarios:}

El Tiempo, domingo 14 de enero de 2001. «Vice: El Manglar está de fiesta».

Correo, domingo 24 de marzo de 2002. «Curso de proyectos de ecología en manglares».

El Tiempo, domingo 12 de enero de 2003. «Manglares de San Pedro: el último refugio del Pacifico sur».

Correo, lunes 13 de enero de 2003. «Festival del Manglar fue un éxito».

El Tiempo, lunes 13 de enero de 2003. «Vice y su Festival del Manglar».

El Tiempo, jueves 08 de enero de 2004. «Inauguran Festival Regional del Manglar. Buscan recuperar humedales de San Pedro y Laguna de Ñapique».

Correo, jueves 08 de enero de 2004. «Inauguran Festival Regional del Manglar».

El Tiempo, domingo 21 de marzo de 2004. «Investigan matanza de flamencos».

\section{Festival Regional Del Manglar}

Durante todas las versiones del Festival Regional del Manglar se han desarrollado, entre otras actividades, las llamadas «ecoconferencias», que han servido para difundir la riqueza biológica que existe en el manglar; la peculiaridad es que, en cada versión de este festival, se daban a conocer nuevos registros para el manglar, ya sea de animales o plantas, en las cuales hemos venido participando.

\section{INTERNET}

Existe el referente de los cursos de capacitación que se realizaron como parte de los Festivales Regionales del Manglar y los pueden ver en:

$<$ http://www.geocities.com/cursohumedales/>. <http://www.geocities.com/curso_aves/>.

$<$ http://www.cifaeci.org.co/biblioteca/bbabr03.htm>.

\section{Foros Científicos}

Expositores en el curso internacional de formación de formadores: «Gestión y conservación de los recursos genéticos forestales». Organizado por la Agencia Española de Cooperación Internacional (AECI), el Instituto Nacional de Investigación y Tecnología Agraria y Alimentaria (INIA-España) y el Centro de Formación de la Cooperación Española de Colombia. Celebrado en Cartagena de Indias-Colombia, del 30 de marzo al 04 de abril de 2003.

Expositores en el II Congreso de la Conservación de la Biodiversidad en los Andes y la Amazonía, y IV Congreso Ecuatoriano de Botánica llevados a cabo en la Universidad Técnica Particular de Loja, Ecuador, del 25 al 29 de agosto de 2003.

Conferencistas en el II Congreso Internacional de Estudiantes de Ciencias Biológicas y IV Congreso Nacional de Estudiantes de Biología, en el Curso Precongreso «Gestión, Conservación y Manejo de Aves Costeras Migratorias del Perú». Organizado por la Universidad Nacional de Trujillo y la Facultad de Ciencias Biológicas, con un total de 30 horas académicas, desarrollado en Trujillo-Perú, del 10 al 12 de octubre de 2003.

Conferencistas en el Seminario Binacional de Manglares: «Promoviendo el intercambio de experiencias en manejo sostenible de los manglares fronterizos Peruano-Ecuatorianos». Organizado por la Fundación Peruana para la Conservación de la Naturaleza (PRO NATURALEZA); la Fundación Ecológica Arco Iris del Ecuador; La Agencia Española de Cooperación Internacional (AECI) y la Fundación CIDEAL del Ecuador, durante los días 13 y 14 de noviembre de 2003, en la ciudad de Tumbes, Perú.

Conferencistas en el X Congreso Nacional de Botánica. V Simposio Nacional de Etnobotánica y Botánica Económica y I Reunión de la Asociación Peruana de Herbarios (APH). Organizado por la Facultad de Ciencias Biológicas y el Herbarium Truxillense de la Universidad Nacional de Trujillo, desarrollado en Trujillo, Perú, del 02 al 05 de mayo de 2004.

Conferencistas en el I Festival del Bosque Seco. Por el Día Mundial del Ambiente. Organizado por la Municipalidad Distrital de Vice y la Asociación Sonqo Llanta Peruano-Suiza. Desarrollado en el distrito de Vice (Sechura, Piura, Perú), el 05 de junio de 2004. 